\title{
Robustness of urban public transport networks
}

\author{
S. Tahmasseby \& R. van Nes \\ Transport and Planning, Faculty of Civil Engineering and Geosciences, \\ Delft University of Technology, The Netherlands
}

\begin{abstract}
Analyses of public transport networks are usually based on a deterministic point of view: it is assumed that all components of the system perform as planned. In reality, however, there are many disturbances influencing public transport services such as variation in demand, service provision, and infrastructure availability. The question is how these disturbances affect the quality of the transport services. As a first step in answering this question a model has been developed that describes the consequences for operators and travellers of variation in infrastructure availability due to disturbances such as incidents, weather, and road works. The model describes the way events might affect infrastructure availability, the resulting impact on the public transport service network (detours, cancelled services) and assesses the outcome for travellers and operators in terms of costs. The model is applied to theoretical networks to illustrate the importance of considering such disturbances in the analysis of public transport networks. Furthermore, the analysis provides insight into possibilities to improve the robustness of public transport service networks by adjusting the service network design or by introducing additional shortcut possibilities in the infrastructure network for detours in case of disturbances. Keywords: robustness, public transport networks, infrastructure availability.
\end{abstract}

\section{Introduction}

For the evaluation of public transport networks assignment models are applied using OD-matrices and descriptions of public transport networks. These evaluation models are based on a deterministic perspective: all types of input are assumed to be known exactly and to be constant over time. These are clearly unrealistic assumptions. The demand pattern varies between hours and over days, while transport supply varies as well, either due to all kinds of organisational aspects in a public transport company, or due to changes in the 
quality and availability of infrastructure. Demand and supply in transport systems are in fact stochastic phenomena. The question then is what the influence of this stochasticity is on the assessment of public transport service networks. Whilst for road networks this topic has been researched extensively, for transport service networks, only few studies are available. For airline service networks Barla and Constantos [1] and Hsu and Wen [5] analyse the impact of variation in demand levels. Yin et al [11] present some measures for reliability in public transport networks, while Lam et al [6] describe a public transport assignment model taking into account that the frequency of a line is influenced by dwelling times at stops, which depend on the number of passenger boarding and alighting. For road networks this topic has been studies by among others, Bell [2], Yin and Ieda [10] and Yamada et al [9].

In this paper we address the issue of the availability of infrastructure and its' consequences for travellers and operators in public transport systems. Infrastructure availability might be hampered by for instance incidents, specific weather conditions, or maintenance works. The paper describes briefly the impact of events on infrastructure availability. Next the consequences on public transport service supply are discussed, followed by the way travellers might react. A model is developed and applied for a hypothetical urban tram network, showing the impact of considering infrastructure availability in public transport service network assessment. These analyses show that considering the possibility of infrastructure failure might lead to different optimal service network designs. Furthermore, the impacts of infrastructure backups on transport network reliability are assessed. Finally conclusions are presented and some recommendations for future researches are given.

\section{Events influencing infrastructure availability}

An implicit requirement in public transport network evaluation studies is that the necessary infrastructure is available with appropriate quality. In reality, however, this is not always the case. Public transport services often share infrastructure with other modes, while in the case of dedicated infrastructure there are still many crossings with other traffic that suffer from congestion and accidents. Furthermore, infrastructure needs maintenance, and since it is part of the city it might be affected by other building or maintenance activities for e.g. sewers, cables, et cetera. Weather might also influence infrastructure availability. For instance there might be slippery roads because of black ice, or fallen trees, and broken wires due to heavy snowstorms. Furthermore, events might be correlated: Bad weather might lead to more accidents, Edwards [4]. Some of these events are unpredictable while others such as road works can be planned in such a way that possible negative effects are minimised.

\section{Impact on public transport service supply}

Given these events that may affect infrastructure availability, the question is how these events might influence the public transport services. Basically, there are two types of effects: 
- Part of the services is out of order (e.g. the scheduled services is limited to a shorter route or might even be split into two different route parts, depending on the location of disturbances).

- Detours are implemented. Transport services will be maintained by diverting the route using the available infrastructure.

In general a public transport service provider will try to maintain the services as good as possible. As such, it is likely that they will attempt to provide detours. Only in the case that no detours can be provided, services will be skipped from the schedule, for instance in the case of multiple disturbances along a line, or in the case of specific weather conditions.

Applying detours can affect the operational costs for public transport operators. Detours require additional driving time and thus additional operational costs. The actual impact, however, might be limited if the extra time is less than the scheduled buffer time for the public transport service involved. Skipping services usually do not affect the operational costs.

\section{Consequences for travellers}

Travellers who are confronted with unexpected changes in public transport service supply usually have limited possibilities to change their behaviour. In the case of detours they will have to accept the additional travel time. Even if alternative paths might be available, it is questionable whether travellers would have sufficient information to consider those alternatives on time. Since these alternatives were not chosen on first hand, it is probable that they will lead to longer travel times. In both cases travellers will thus experience an additional travel time, which due to the uncertainty involved will weigh more in their appreciation than standard in-vehicle time. Please note that proper information supply might reduce the uncertainty and thus the weight for additional travel time. Whilst normal travel costs $(€)$ will be:

$$
C_{t n}=\sum_{i=1}^{O} \sum_{j=1}^{D} P^{n}{ }_{i j} \bullet T_{i j} \bullet V O T_{1}
$$

where:

$P_{i j}^{n} \quad=$ number of trips between origin $i$ and destination $j$

$T_{i j} \quad=$ travel time between origin $i$ and destination $j$ via shortest path (hr)

$V O T_{1}=$ value of time $(€ / \mathrm{hr})$

The additional travel cost $(€)$ due to additional travel time using detours will be:

$$
C_{t e}=\sum_{i=1}^{O} \sum_{j=1}^{D} P_{i j}^{d} \cdot\left(T_{i j}^{d}-T_{i j}\right) \cdot V O T_{2}
$$

where:

$T_{i j}^{d}=$ travel time due to using detours (hr) 
$P_{i j}^{d}=$ number of trips using detours between origin $i$ and destination $j$

$V O T_{2}$ is assumed to be larger than $\mathrm{VOT}_{1}$ because of uncertainties for passengers.

In the case of cancellation runs, it might be impossible for travellers to reach their destinations by public transport. The cost $(€)$ due to trip cancellation is:

$$
C_{t c}=\sum_{i=1}^{O} \sum_{j=1}^{D} P_{i j}^{c} \cdot C_{c}
$$

where:

$P_{i j}^{c}=$ number of cancelled trips between origin $i$ and destination $j$

$C_{c}=$ penalty cost of trip cancellation $(€)$

Hence, the total travel cost consists of normal travel cost and additional travel cost due to using detours or trip cancellation in a year yields:

$$
C_{t}=C_{t n}+C_{t e}+C_{t c}
$$

Summarising, there are two main types of indicators for events that influence infrastructure availability:

- Additional travel time;

- Number of cancelled public transport trips.

The first might be classified as travel time reliability, while the second relates more to the robustness of the service network, i.e. the ability to perform its function as a transport system.

\section{Modelling framework}

The model presented in this paper is focused at getting a first estimate of the impact of such events on network performance. Therefore, a level of detail is chosen such that the mechanisms described in the previous sections are dealt with.

The model assumes a predefined infrastructure network. The public transport service network is defined as a set of lines using the infrastructure network given a budget for operating the services under regular conditions. The level of demand is assumed to be independent of the quality of the services offered.

In the first stage the simulation of events for a certain period of time, e.g. a year is taken into account. Typical events that are considered are: incidents, road works, bad weather consisting of storm, black ice, snow, and thunderstorm. The events are assumed to be independent of each other. The frequency and duration of each type is based on realistic estimates or on empirical values. Please note that multiple events might take place at the same time. Each period in which no new events start or end is called a state.

The second stage determines which links of the infrastructure network are affected by the events. Depending on the type of events one or more links might fail, that is being not available for use by public transport services. A MonteCarlo approach is used to select failing links, while for simplicity sake no correlation between failing links is assumed. 
The third stage considers the consequences for public transport service supply. For each state, depending on the type of event that is applicable, the following possibilities are considered:

- Detours

- Skipping runs for a part of a line: The remaining parts operate as planned;

- Skipping a set of runs of a line;

The fourth stage considers the consequences for travellers for each state. The reference in this case is the travel time based on the shortest path in the condition without disturbances. The travel time includes waiting time and in-vehicle time. In the case of detours travellers are assumed to accept the additional travel time. In the case that the additional travel time becomes too long compared to the original travel time, e.g. twice as long, it is assumed that travellers cancel their public transport trip. If it is no longer possible to travel by public transport because runs are skipped or stops are not served, the corresponding public transport trips are cancelled as well.

\section{Application of the model to a hypothetical network}

The model presented in section 5 is applied to a hypothetical urban public transport system. The city has a ring-radial structure having the city centre in the middle. The city is served by a tram network. The transport system consists of eight radial lines. In addition to radial lines, there are possibilities to apply different either ring lines or ring infrastructures. To distinguish between these two, we here study two main cases as follows:

A. Ring infrastructure is used by a service line in the network

B. Ring infrastructure is only used as a backup to provide detours in case of blockage in original lines of the network

In the latter case, we consider a fixed public transport service network consisting of eight radial lines. In order to increase the reliability of the service network, extra links have been built. In the case of a tram system this requires rail infrastructure with interlock switches et cetera. When a part of the original route of a radial line fails, the service might diverge from its original route using the extra infrastructure links.

In terms of network configurations, the following combinations are studied:

1 Radial lines

2 Radial lines and centre ring

3 Radial lines and small ring

4 Radial lines and large ring

5 Radial lines and outer ring

Figure 1 illustrates variant 3 in which the ring facility used by a service line (case A). The dashed lines indicate the location of the rings for the other variants. Obviously in variant 1 (no ring) there is not any ring infrastructure. As a result it can be expected that variant 1 will be quite sensitive for failures in infrastructure availability as there are no possibilities for detours. Depending on the location of the ring, the other variants will be more robust. Furthermore, the infrastructure 
costs are not equal for all variants. A detour for a radial line uses a part of the ring to switch to a neighbouring radial. In the case of ring lines only detours via the city centre are possible. The operational budget is identical for all alternatives. As a result the frequencies are highest for variant 1 and lowest for variant 5 in case A. In case B in which ring facilities are only used as a backup, there is no difference for service frequency between variants.

The analysis consists of 20 simulations for a period of a year. The selection of failed links always relates to all 64 links in the network. As a result it might be that in certain states only links fail that are not in use by the public transport service network for that variant. As such the network of variant 1 has the advantage that less infrastructure links are used. Travel time is translated into costs using a value of time of $€ 10$ per hour. The value of time for additional travel time is twice as high ( $€ 20$ per hour), while the penalty for cancelled public transport trips is $€ 20$ per trip.

\subsection{Results}

Due to the indicated events there are about 7 failures per link per year. The total out of order time for a link due to such a set of events is 1.6 days per year on average. Concerning operational costs, the total additional operational costs, seem negligible. The maximum is less than $0.06 \%$ of the total annual operational costs. The small amount of additional operation costs is due to the short period of disturbances leading to long detours compared to the total annual operation costs.

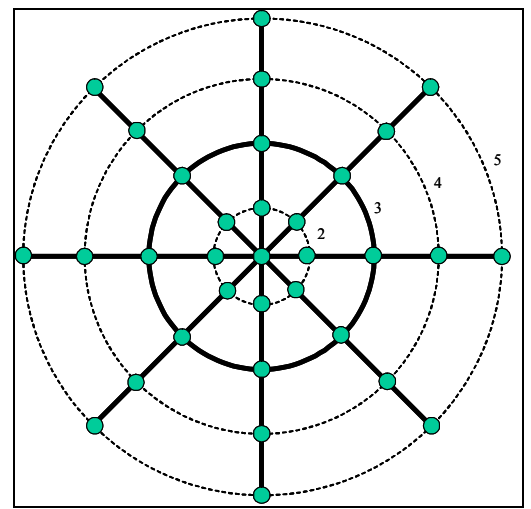

Figure 1: Lay-out of the infrastructure network for variant 3: radials and small ring (dashed lines indicate the infrastructure for the other variants).

In terms of effects on infrastructure availability, there appears to be a limited number of cases where it is possible to provide detours. In all other cases no alternative route is available, implying that in many cases runs need to be skipped. 
For the network assessment, the following results will be discussed:

- Network reliability (travel time reliability and robustness)

- Total network costs

\subsubsection{Network reliability}

Network reliability is assessed by extra costs due to detours and the costs for trip cancellation (section 4). Figure 2 shows these costs for case A in which ring lines are operated.

Using detour leads to additional travel time and thus additional travel costs. For variants 1 no detours are possible, therefore there is no additional travel time. For the subsequent variant the additional travel time increases from $0.04 \%$ for variant 2 to $0.39 \%$ for variant 5 . Variation in travel time due to failures in infrastructure availability is thus limited in importance, although a systematic trend can be noted. Trip cancellation costs add up to more than $7 \%$ of the travel costs in regular conditions in variant 1 . The consequences of the events decrease per variant, having a minimum for variant 4 . For variant 5 there is a slight increase in additional costs due to the larger detours in this variant. These results clearly indicate that different network structures strongly influence the reliability of transport service networks.

For case B in which ring infrastructure is used only as a backup, trip cancellation cost does not change significantly for each variant compared to case A. However, additional travel costs for variant 4 and variant 5 rise slightly. This is due to shorter travel times under regular conditions in the corresponding variants.

Please note that in regular conditions, travel times in variants having ring line services might be longer than travel times for variants having only radial lines, due to the given constraint of a constant operational budget. In general, the aforementioned outcomes clearly indicate that having ring infrastructure backup increases the reliability of transport service networks.

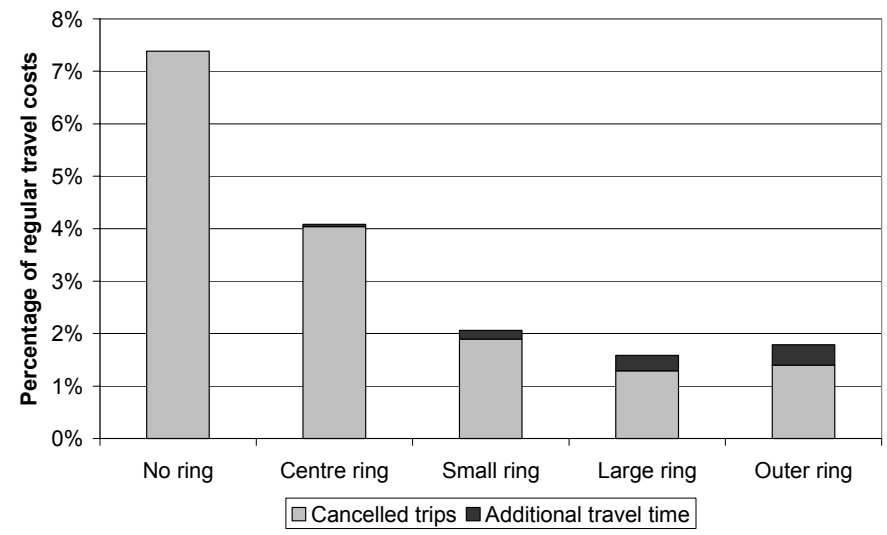

Figure 2: Additional travel costs due to events as a percentage of the travel costs in regular conditions (case A). 


\subsubsection{Total network costs}

In this section a comparison is made of the total network costs for all five variants. The total network costs include total travel costs including reliability costs, total operational costs, and infrastructure costs. Infrastructure costs include investment and maintenance costs, which are based on data of the Ministry of Transport, Public works and Water management [7]. Comparing case A and case $\mathrm{B}$, due to the fact that supplementary infrastructure is used only as a detour in disturbed conditions in case $\mathrm{B}$, the corresponding yearly maintenance costs are less than for regularly used infrastructure as in case A. Thus the yearly maintenance costs are estimated as $3 \%$ of the investment costs for case A and $1 \%$ for case B. These lead to 0.23 million Euros per kilometer as annual costs for one direction in case A and 0.18 million Euros per kilometer for case B. In order to have an outlook of total network costs' components, total network costs consist of $65 \%$ travel costs, $23 \%$ operational costs, and $12 \%$ infrastructure costs for variant 1 in case A under regular conditions.

In order to show the relevance of considering infrastructure availability, a distinction is made between the option under regular conditions and the option including events affecting infrastructure availability. For case A, variant 1 under regular conditions is used as a reference base. Figure 3 shows the performance for all five variants for both conditions in case A. In regular conditions, variants 1 and 2 have a nearly identical score. For the other variants the impact of lower frequencies and higher infrastructure costs leads to higher total network costs. However, if infrastructure availability is considered variant 2 appears to be the most attractive variant. Considering travel time reliability and robustness characteristics thus affects network type choice.

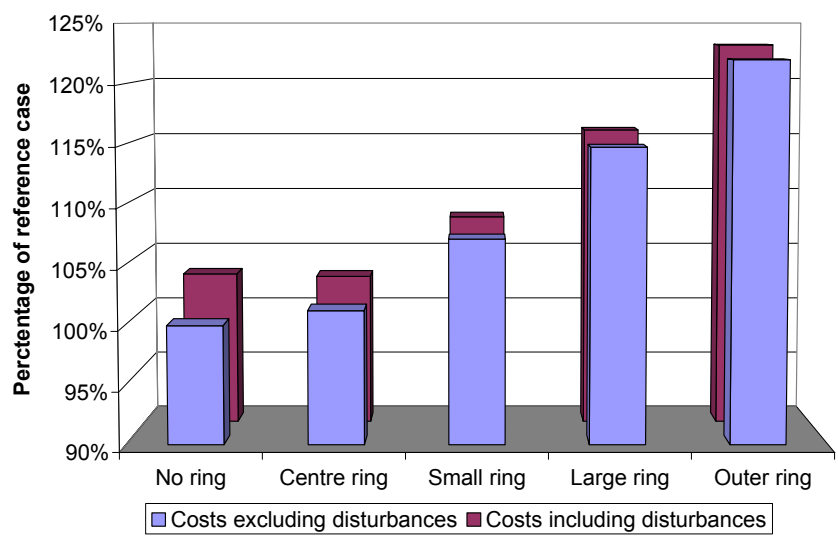

Figure 3: Total network costs as percentage of the reference base for case A.

In case $\mathrm{B}$, again a comparison is made of the total network costs for all five variants. Here, the operational costs relate to radial lines and their detours. Results are shown in figure 4 . Like before, variant 1 under regular conditions is 
used as a reference base. Results show that again variant 2 (involving centre ring infrastructure) is the best option in terms of total network costs. With increasing ring infrastructure length the total network cost increases accordingly.

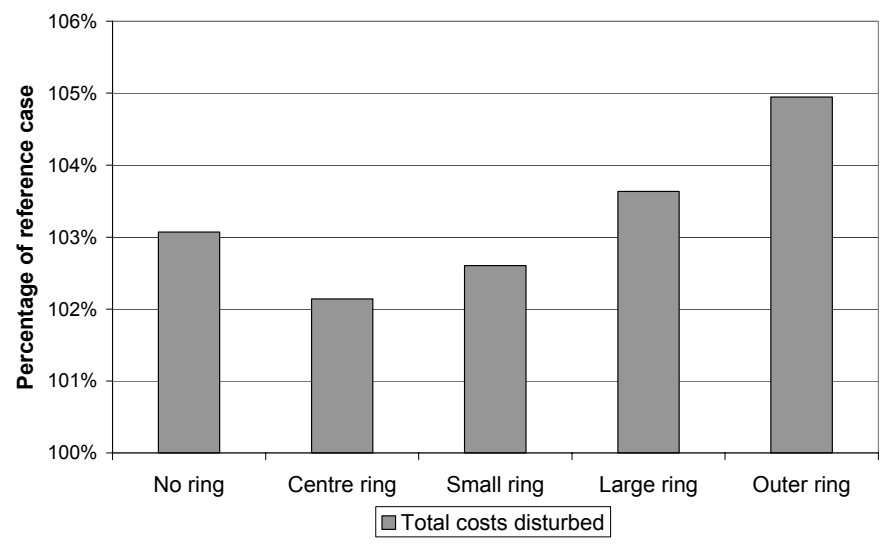

Figure 4: Total network costs as percentage of the reference base for case B.

\section{Conclusions and recommendations for research}

The analysis with the newly developed model showed that accounting for variation in infrastructure availability has a clear impact on total travel costs (more than 7\%), while having a minor impact on operational costs. Comparison of network variants showed that depending on the criterion used different network types might be preferred. A focus on robustness, that is limiting the number of cancelled public transport trips, would favour network variants having sufficient detour facilities such as the ring radial networks having a large ring or an outer ring. A more balanced approach in which the total network costs, i.e. total travel costs, total operational costs and infrastructures are considered, leads to the choice for a network having a centre ring, while in the case that no infrastructure failures are considered a network having radials only would be equally attractive. Of course, the model and the analysis presented in this paper is a first step towards a more complete approach considering all kinds of stochasticity on network assessment and thus network design.

The framework presented in this paper can be extended in various ways. More types of events might be considered such service failures, e.g. vehicle breakdowns, or substantial variations in demand levels and patterns. Furthermore, correlations between events might be included (e.g. the impact of bad weather on incidents as well as demand). Moreover, long-term choice behaviour of travellers might be included as well since a network design also implies a certain level of reliability. Finally, the extended model might be applied for realistic case studies such as the tram networks of The Hague and Amsterdam. 


\section{Acknowledgement}

This study is supported by the Transport Research Centre Delft and by the BSIKprogramme Next Generation Infrastructures.

\section{References}

[1] Barla P. \& Constantatos, C., Airline network structure under demand uncertainty, Transportation Research E 36, pp. 173-180, 2000

[2] Bell, M.G.H., A game theory approach to measuring the performance reliability of transport networks, Transportation Research B34, pp535545,1999

[3] De Jong, P., Towards robust public transport networks, MSc-Thesis, Transport \& Planning, TU Delft, Delft (in Dutch), 2005

[4] Edwards, J.B., The temporal distribution of road accidents in adverse weather, Meteorological Applications 6, 59-68, 1999

[5] Hsu C.I. \& Wen, Y.H., Reliability evaluation of airline network design in response to fluctuation in passenger demand, Omega 30, pp. 197-213, 2002

[6] Lam W.H.K., Zhou, J. \& Sheng, Z., A capacity restraint transit assignment with elastic line frequency, Transportation Research Part B 36, pp. 919-938, 2002

[7] Ministry of Transport, Public Works and Water management, AGV, Vrije Universiteit Amsterdam, Handboek economische effecten infrastructuur, Den Haag, 1996

[8] Van Nes, R., Design of multi-modal transport networks: A hierarchical approach. Trail Thesis Series T2002 / 5, DUP, Delft, 2002

[9] Yamada, T., Yoshimura, Y. \& Mori, K., Road network reliability analysis using vehicle routing and scheduling procedure, in (ed) Taniguchi, E., R.G. Thompson, Logistic systems for sustainable cities, Pergamon, 2004

[10] Yin, Y. \& Ieda, H., Optimal improvement scheme for network reliability, Transportation Research Record 1783, pp. 1-6, 2002

[11] Yin Y., Lam, W.H.K., \& Miller, M.A., A simulation-based reliability assessment approach for congested transit networks, Journal of Advanced Transportation 38, No. 1, pp. 27-44, 2003 